\title{
Linx
}

Revue des linguistes de l'université Paris X Nanterre

$58 \mid 2008$

Aspects de comme

\section{Le mot comme : problèmes et perspectives en synchronie}

Antoine Gautier

\section{OpenEdition}

Journals

Édition électronique

URL : http://journals.openedition.org/linx/325

DOI : $10.4000 / \operatorname{linx} .325$

ISSN : 2118-9692

Éditeur

Presses universitaires de Paris Nanterre

Édition imprimée

Date de publication : 1 juin 2008

Pagination : 13-23

ISSN : 0246-8743

\section{Référence électronique}

Antoine Gautier, «Le mot comme : problèmes et perspectives en synchronie », Linx [En ligne],

58 | 2008, mis en ligne le 15 février 2011, consulté le 30 avril 2019. URL : http://

journals.openedition.org/linx/325; DOI : 10.4000/linx.325

Département de Sciences du langage, Université Paris Ouest 


\title{
Le mot comme : problèmes et perspectives en synchronie
}

\author{
Antoine Gautier \\ Paris IV - Sens, Texte, Histoire
}

Les éléments de synthèse qui suivent se proposent d'offrir une lecture globale des derniers travaux sur le mot comme en soulignant les grandes problématiques qu'a favorisées le récent regain d'intérêt pour ce morphème. Cet état de l'art se veut un prolongement de la présentation de Flaux \& Moline (2008), axé en priorité sur les problèmes méthodologiques que soulève l'étude particulière du morphème comme.

On observera d'abord que les études sur comme adoptant une perspective morphosyntaxique traditionnelle se heurtent à un problème qui est d'abord taxinomique : dans quelle(s) catégorie(s) grammaticale(s) faut-il classer ce mot? Par ailleurs, doit-on postuler que la pluralité des emplois de comme correspond aux facettes d'une même entité lexicale (hypothèse polysémique) ou bien à des unités distinctes (hypothèse homonymique)? Et quels sont du reste les présupposés et les implications de ces différentes hypothèses? Ce sont là les problèmes que doivent résoudre les études grammaticales et linguistiques du mot comme - quitte à ménager de nouvelles places dans les nomenclatures.

D'une part, dans des travaux relevant essentiellement de la sémantique ou de la stylistique, comme est souvent évoqué dans les champs onomasiologiques de la manière, du degré et de la comparaison. L'un des attraits de ces approches est de dégager la valeur de comme par opposition à celle de morphèmes sémantiquement voisins ou concurrents formant système; mais à leur tour les notions de manière, de degré et de comparaison appellent un effort définitoire. D'autre part, un second ensemble de contributions cherche explicitement à pallier l'un des défauts majeurs de l'approche 
sémasiologique - qui est aussi son principal atout, à savoir l'adoption d'un simple signifiant pour point de départ. Ces travaux s'attachent à distinguer les propriétés qui sont attribuables au seul morphème, c'est-à-dire à un élément objectivé et isolé de tout contexte, et celles qui ne le sont pas, étant au contraire liées à ce qu'on peut appeler des «effets contextuels». Cela peut en particulier conduire à l'identification de constructions, dotées de propriétés non compositionnelles, par conséquent non observables à partir du seul morphème comme.

\section{I. À quelle(s) catégorie(s) appartient comme?}

Les approches descriptives traditionnelles ${ }^{1}$ ont pour objectif de consigner dans une nomenclature les différents éléments de la langue et non de discuter la constitution de cette nomenclature. Pour un mot tel que comme, qui connait comme on sait une large variété d'emplois, on note d'importantes variations dans le classement que proposent les grammaires, mais aussi quelques positions communes.

Ainsi, dans le corpus examiné, la plupart des auteurs reconnaissent un avatar conjonctif de comme, porteur de trois valeurs majeures : comparative, temporelle et causale. En confrontant ces trois valeurs, certains remarquent que la confusion fréquente des deux dernières est sans doute liée à ce que l'effet de sens causal procède de la valeur temporelle, qui note l'antériorité ou la simultanéité ${ }^{2}$. Sandfeld (1965: 325) reconnaît pour sa part qu'il «n'est pas toujours possible de faire nettement la différence entre comme causal et comme temporel», et il réunit ces deux valeurs autour d'un comme "primitivement [...] comparatif», qui peut aussi marquer la simultanéité (de même que quand et lorsque) et «la conformité de la cause avec l'effet» (à l'instar de puisque). En comparant ainsi plusieurs morphèmes, Sandfeld note un fait de système rarement souligné, à savoir que la valeur temporelle de comme remédie à l'impossibilité pour quand de fonctionner avec l'imparfait sans se charger de facto d'une valeur itérative (Ex. : *Quand il sortait, il se mit à pleuvoir).

Malgré tout, il n'existe qu'un consensus limité autour de comme conjonctif. L'édifice semble même se démanteler de part et d'autre: non seulement la relation entre les valeurs conjonctives n'est pas régulièrement pointée ${ }^{3}$ (il y aurait donc plusieurs conjonctions), mais en outre la catégorie de comme pour chaque valeur ne fait pas l'unanimité : ainsi pour Wagner \& Pinchon (1993 : 451, 656), comme comparatif est selon sa « portée » tantôt conjonction, tantôt adverbe. Lorsqu’il est question des segments comparatifs non propositionnels introduits par comme, les points de désaccord sont bien connus : ils touchent à l'alternative entre une interprétation par l'ellipse et le

\footnotetext{
${ }^{1}$ L'babitus des «grammaires traditionnelles » se compose d'un certain nombre de traits réguliers: observation exclusive des réalisations écrites et littéraires de la langue, caractère normatif, absence de cadre théorique de référence, travail descriptif et taxinomique ne distinguant pas les niveaux d'analyse (Lerot apud Lauwers \& Neveu, 2007 : 13).

${ }^{2}$ C'est ce que défendent notamment certaines grammaires de notre corpus, telles Arrivé et al. (1986 : 110) ou Gardes-Tamine (1998: 52).

${ }^{3}$ Grevisse (1993 : 1626 sqq., \$1078-1079) distingue trois propositions adverbiales en comme sans lien entre elles.
} 
postulat d'un comme préposition. Si Monneret \& Rioul (1999) s'élèvent contre cette dernière hypothèse, celle-ci est soutenue par Moignet (1981), Béchade (1996: 296), Wilmet (1997) et (quoique prudemment) par Riegel et al., (1994: 515). Quant au Bon Usage, des cas tels que «Je le prends comme témoin » contraignent ses auteurs à délaisser le rasoir d'Occam pour le marteau du forgeron et à créer ex nibilo une classe d'introducteurs ${ }^{4}$.

Cela dit, les études récentes proposent des tests formels convaincants, qui mettent en évidence des propriétés prépositionnelles du mot comme dans quelques cas précis (Pierrard, 2002) : (i) les constructions à attribut du complément d'objet («Je considère Paul comme fou. ») et (ii) les segments périphériques en comme $\mathrm{N}$ introduisant une prédication seconde ("Tu prendras quoi/ du chocolat comme dessert ?»). Le principal critère est que la reconstruction d'une configuration comparative est plus difficile à partir de ces exemples qu'à partir des comparants non propositionnels ${ }^{5}$; mais encore faut-il prêter crédit au test de la reconstruction de séquences absentes en surface (Desmets, $2001: 144)$.

Autre point commun des ouvrages examinés (relevant ou non de la «tradition»), la reconnaissance du comme adverbe exclamatif, qui ne soulève guère de problème dans le cas de l'exclamation directe : «Comme c'est beau! ». On sait pourtant en quels termes la classe grammaticale de l'adverbe a été décrite, ce qui a amené à distinguer une fonction adverbiale non coextensive à la classe proprement dite ${ }^{6}$ (Nølke, 1993 : 25). Dans l'exclamation, le morphème est associé au haut degré par Riegel et al., à l'intensité par Wagner \& Pinchon ou Charaudeau (1992: 369), lequel reprend l'idée culiolienne de «comparaison non évaluable tant elle est extrême». Comme est généralement analysé dans ces cas-là comme un adverbe portant sur des procès ou des « qualités » (Arrivé et al., 1986 : 263). Mais le consensus sur ces emplois s'effrite dès qu'on approche de l'interrogation et de l'exclamation indirectes, comme étant alors donné pour équivalent de combien ou comment, selon les cas. Ainsi Wagner \& Pinchon (1993 : 441) voient dans « Vous n’ignorez pas comme vont les choses » une persistance archaïque du «Comme est-il mort? ? de Corneille (où comme équivaut bien à comment), mais ils font correspondre comme à combien dans un tour moderne qu'ils décrivent comme une interrogation portant sur la manière : «Avez-vous vu comme il dort? ». Le Bon Usage, pour sa part, fait de comme un adverbe équivalent « littéraire » de comment dans l'interrogation indirecte: "Vous verrez comme il faut que l'on gouverne » (Grevisse, 1993: 1390, \$940) mais reconnait ailleurs l'exclamation indirecte dans «Regarde comme il est sage » (Id. : 1681). Pour Sandfeld (1965: 60), comment est limité, dans l'exclamation, à l'expression de la manière, autrement dit-il caractérise la qualité

\footnotetext{
${ }^{4}$ Défini comme un "mot invariable qui sert à introduire un mot, un syntagme, une phrase» (Grevisse-Goosse 1993 : 1558 sqq., \1043-1044).

5 Comparer « Je l'ai aimée comme [aime] un fou» et «*Je le considère comme [je considère] (un) fou ».

${ }^{6}$ Chez G. Guillaume, les classes de parties de langue se définissent par la prévision de leur mécanisme d'incidence. La distinction entre catégorie et fonction se trouve donc partiellement revue : l'adverbe, en l'occurrence, se définit par son incidence externe du second degré, autrement dit par le fait qu'il constitue un apport de sens à un autre apport de sens, par exemple de l'adjectif au nom (un enfant très pâle) ou du verbe au nom sujet (il dort beaucoup) (Moignet 1981: 50 sqq.). On peut voir dans cette incidence le trait fondamental de la fonction adverbiale.
} 
« remarquable, excellente, révoltante, blâmable, etc. » d'un procès, d'un état ou d'une qualité ; à l'inverse, comme traduit principalement le degré, mais il peut aussi apparaittre comme un équivalent familier de comment: «Ça m’épate comme vous dirigez. » On peut tenter d'expliquer la disparité de ces multiples analyses; il ressort tout d'abord que l'objet de la demande d'information ou de l'exclamation n'est pas toujours bien identifié dans les phrases complexes à Interrogative ou Exclamative enchâssées. En outre, le sens très ténu de comme lui confère une place ambiguë entre exclamation et interrogation (avec circonspection, Riegel et al., 1994: 404, en font mention). Seul Sandfeld semble tenir compte de la porosité de la frontière entre les deux «modalités». Il affirme ainsi «qu'une proposition interrogative indirecte, introduite par un mot interrogatif marquant le degré ou la quantité, s'emploie également comme exclamation »; cependant, cette analyse ne semblera pas valable au même degré pour «Si c'est possible de rentrer à pareille heure !» et «Ah mon frère, comme tu as raison et que c'est vrai !», que Sandfeld (1965: 78) range pourtant dans la même catégorie. En tout état de cause, l'analyse des interrogatives indirectes est grandement compliquée par l'instabilité actuelle des mots comme, comment et combien, dont les recouvrements, les concurrences et l'usure sont encore difficilement lisibles, étant en outre soumis à d'importantes variations sociolectales.

Autre valeur du comme adverbe, l'emploi modalisateur ${ }^{7}$ est reconnu par la plupart des auteurs ${ }^{8}$ mais il connaît des analyses très différentes sur le fond. Certains, à l'image de Wagner \& Pinchon, soulignent une valeur d'approximation ou parlent « d'adverbe modificateur » (Riegel et al., 1994 : 515), mais d'autres le rattachent explicitement à la comparaison (Charaudeau, 1992: 369; Monneret \& Rioul, 1999: 267). Pour ces derniers, le lien avec les emplois comparatifs s'établit en restituant le terme implicite d'une relation d'analogie :

Il se tut encore quelques instants (pour une certaine raison), comme (s'il se taisait parce qu'il était) désireux de plonger plus avant on comme (s'il était) désireux... (Monneret \& Rioul, ibid.)

Paradoxalement, le rattachement au système comparatif ne s'accompagne cette fois d'aucune interrogation sur la catégorie grammaticale de comme: sa nature d'adverbe n'est pas mise en doute.

Au-delà de ces emplois bien observés, on rencontre dans les grammaires quelques cas moins communs. Arrivé et al. (1986: 22), Monneret \& Rioul (1999) et Béchade (1996) recensent ainsi un comme de coordination (explicitement conjonctif ou non); si les premiers s'appuient sur des critères de distribution et d'accord («L'alcoolisme comme le tabagisme sont des fléaux sociaux. », Arrivé et al., $1986: 25)$, Monneret \& Rioul (1999 : 261) n'évoquent la coordination qu’à demi mot, comme un effet secondaire de «l'expression de la similitude, réduite au minimum». Or, il semble

\footnotetext{
${ }^{7}$ Opérant par exemple sur un attribut : «J'en suis resté comme abasourdi » (Riegel et al., 1994 : 515) ou sur un adjectif apposé : «Il se tut encore quelques instants, comme désireux de plonger plus avant» (Monneret \& Rioul, 1999 : 267).

${ }^{8}$ Gardes Tamine (1998 : 76), Riegel et al. (1994 : 515), Monneret \& Rioul (1999).
} 
qu'une approche strictement syntaxique du phénomène soit préférable à une analyse fondée sur le degré d'analogie de deux prédications, lequel n'est pas toujours facile à quantifier (voir la contribution de Mouret \& Desmets dans le présent numéro).

Comme on peut le constater, les cadres usuels de la morphosyntaxe semblent peu préparés à intégrer de manière cohérente tous les emplois de comme. Moins assujettis à cette vulgate grammaticale, Damourette \& Pichon (1941-1943) proposent une approche originale du morphème dont certains aspects seront repris sous la plume d'autres linguistes travaillant la même matière (notamment Le Goffic). Selon leur analyse, les emplois de comme sont répartis dans trois catégories : les chainons à noyaux factifs $(\$ 3122)$, les chaînons à noyaux non factifs $(\$ 3123$ à 3127), et les emplois exclamatifs $(\$ 3128)$. Les premiers et deuxièmes correspondent aux occurrences de comme introduisant un segment subordonné, propositionnel ou non. Pour ces auteurs, c'est la valeur «confrontante» (i.e. comparative) qui est primordiale. Dans les segments propositionnels, où comme est de nature conjonctive, elle subsume les valeurs comparative, temporelle et causale (ce qui est un avis assez partagé, on l'a vu). Dans les chainons à noyaux non factifs, Damourette \& Pichon subsument sous la valeur confrontante les tours échantillants (i.e. comparatifs elliptiques) et qualifiants, considérés comme très proches, ainsi que les tours quasiceptifs. On note par ailleurs que ces derniers reçoivent la même analyse chez Monneret \& Rioul (1999). Étudié à part, l'emploi exclamatif est dérivé d'anciennes constructions interrogatives directes ou indirectes, considérées par les auteurs comme disparues: "Comme est-ce que chez moi s'est introduit cet homme ?» (Molière, Ibid. : \3128); "Il commença à lui raconter comme il avoit entendu sa conversation... » (Mme de Lafayette, Ibid. : \3128).

\section{Approches sémasiologiques}

N'hésitant pas à se défaire de l'appareil traditionnel de la morphosyntaxe, la tentative de description unifiée du morphème comme menée par Damourette et Pichon a été pionnière dans un domaine aujourd'hui plus fréquenté. Depuis lors, en effet, un certain nombre d'études linguistiques ont été exclusivement consacrées à comme. Adoptant une démarche sémasiologique, ces études avaient en commun le postulat d'un unique morphème polysémique9 correspondant à la pluralité des emplois et d'effets de sens rencontrés en discours.

C'est le cas chez Moignet (1981), qui, par le biais du concept de subduction (éso- et exotérique), étend son analyse à un micro-système composé de comme et ses dérivés étymologiques combien et comment. Il répartit l'ensemble des emplois du mot sur les catégories de l'adverbe non-prédicatif ${ }^{10}$ et de la conjonction. La première rassemble les emplois exclamatifs, comparatifs et interrogatifs, la seconde les emplois temporels et causaux. Mais Moignet n'exclut pas, dans la première catégorie, que comme

\footnotetext{
${ }^{9}$ Mais Gardes-Tamine (1998 : 75) d'une part, et Desmets (2001 : 54) d'autre part, ont pu faire état de comme homonymes.

${ }^{10}$ C'est-à-dire d'adverbes dont le contenu sémantique est ténu et/ou abstrait: au contraire de courageusement, qui est prédicatif, ainsi ou ailleurs ont des signifiés «dématérialisés » (Moignet, 1981 : 196).
} 
comparatif ait des propriétés de relatif sans antécédent («Il ment comme il respire », Ibid. : 198) voire de «quasi-préposition, du fait que la phrase qu'il introduit est très aisément elliptique du verbe » (Ibid. : 198). La démarche critique de Le Goffic (1991, 1993, et passim) conduit elle aussi à une refonte de certaines catégories grammaticales, et par conséquent à un réagencement des emplois de comme. Dès ses premiers travaux touchant au problème, Le Goffic (1991) fonde son analyse sur l'hypothèse de la famille des mots * kw- dans laquelle celui-ci assigne à l'adverbe tous les emplois de comme ; il y associe selon les cas le rôle de connecteur intégratif, qui lui confère le rôle de subordonnant et souligne sa double fonction, dans la structure matrice et dans la structure enchâssée :

Le terme connecteur est $[\ldots]$ le pivot organisateur des deux structures de phrase. Il est doté d'une fonction dans sa subordonnée [...]. Mais il est aussi en rapport avec le verbe principal : il a en fait une double portée (fondamentalement à l'identique), sur les deux verbes [...]. (Le Goffic, 1993 : 45)

Autour de cette valeur centrale sont distribuées la plupart des valeurs secondaires (Le Goffic, 1993 : 394 sqq.) associées à des changements contextuels, syntaxiques et sémantiques (ellipse, absence de déterminant du nom situé à droite de comme, etc.). La ligne théorique demeure la même dans l'importante étude de Fuchs \& Le Goffic (2005), qui confère cette fois à comme la valeur fondamentale de marqueur d'identité, opérant tantôt sur des «manières de faire » (modus faciendi), tantôt sur des "manières d'être » (modus essendi). Le choix se porte ainsi sur une base sémantique abstraite dont dérivent dix-neuf types de configurations syntaxico-sémantiques réparties en trois sous-ensembles.

Certains travaux de Pierrard (1999b, 2002a, et passim) et de Léard (Léard \& Pierrard, 2003) adoptent une même approche sémasiologique visant à produire une description unifiée du morphème. Dans la continuité de Le Goffic (1991), Pierrard fait du morphème comme une forme en $Q U$ - dont les emplois essentiels combinent les traits d'indéfinition et d'identité. "Le partage du trait /INDÉFINI/ entre deux prédications, expliquent les auteurs, représente le mécanisme de subordination, d'intégration" (Léard \& Pierrard, 2003 : 207). Reprenant l'esprit du guillaumisme (mais non la lettre), les auteurs donnent le primat au sémantique - sinon dans les faits, du moins dans l'analyse et la typologie.

Dans cette optique, les auteurs dégagent certaines valeurs prototypiques de comme, délimitées par la superposition des traits d'indéfinition et d'identitế ${ }^{11}$; celles-ci sont complétées par des emplois dits "périphériques", qui trahissent un affaiblissement sémantique et ne relèvent, quant à eux, que de l'identité. Soucieux de ne pas objectiver à l'excès (voir infra), les auteurs confrontent chaque valeur à des expressions concurrentes dans un contexte donné, ce qui, notamment, fournit quelques éléments d'une typologie en creux des différents emplois de comment.

Le problème de la catégorisation de comme prend ensuite une nouvelle dimension avec la notion de proforme indéfinie, introduite par les mêmes auteurs afin de répondre à deux questions essentielles :

\footnotetext{
${ }^{11}$ Ce qui peut s'illustrer ainsi : Il ment comme il respire > «la manière (indéfinie) dont il ment est identique à la manière (indéfinie) dont il respire. »
} 
Comment expliquer la large diffusion des termes indéfinis en $q u$ - en connexion interpropositionnelle $[\ldots]$ ? Et comment concilier des emplois particuliers disséminés dans de multiples tours avec une appréhension cohérente de leur rôle dans le mécanisme de subordination? (Pierrard, 2005 : 235)

Ces proformes sont définies par un faisceau de traits assez réguliers (qui peuvent être neutralisés selon les contextes) : au plan sémantico-référentiel, l'indéfinition affectant les traits \pm animé (que, qui), lieu (où), temps (quand), manière (comme, comment), etc.; au plan syntaxique, l'instauration d'un rapport de "dépendance par cosaturation", associé au marquage fonctionnel du rôle joué dans la prédication intégrée (fonction proforme) (Pierrard, ibid. : 239).

Malgré ces propriétés qui l'intègrent de droit à l'ensemble des proformes, comme se distingue de celles-ci par des aspects importants (Pierrard \& Léard, 2004: 270 et sqq.) : en premier lieu, il refuse les emplois interrogatifs, étant remplacé à ce poste par comment; deuxièmement, son sémantisme ne différencie pas toujours la qualité et la quantité; enfin, il ne tolère pas la réduction propositionnelle (Muller, 1996) à la proforme « nue » (ou sluicing) : «*je ne sais pas comme » vs. « je ne sais pas comment».

De toute évidence, la catégorie proforme est d'une grande plasticité. C'est sans doute là, du reste, que réside son principal intérêt : dans la déformabilité acquise par l'appareil descriptif. En effet, l'ensemble des critères définissant la catégorie n'a pas à être intégralement réalisé dans un objet particulier pour qu'il soit intégré à celle-ci. En somme, les problèmes rencontrés par l'approche traditionnelle ont trouvé des amorces de solutions dans l'hypothèse polysémique, qui a permis des descriptions unifiées du morphème via un signifié fondamental associé au signifiant, ainsi que dans la classe des proformes, qui a mis en lumière d'importantes parentés syntactico-sémantiques entre des formes jusque là séparées (parenté confortée par l'étymologie, qui plus est).

\section{Comme : comparaison et approches constructionnelles}

Il est difficile d'évoquer comme sans aborder plus généralement le système de la comparaison (Rivara, 1990). Question très vaste, intéressant autant les sciences du langage que les philosophes, elle ne sera considérée ici que sous son aspect proprement linguistique. Comme processus sémantique, la comparaison pourrait se définir comme le marquage «d'un rapport d'égalité ou d'inégalité d'une action ou d'un fait quelconque avec une autre action ou un autre fait » (Sandfeld, 1965 : 424). Mais le caractère peu approprié de la dyade égalité /inégalité fera préférer la définition de Muller (1996: 89), plus moderne, pour qui la comparaison consiste en une «confrontation de mesures»; ce procédé implique au moins deux quantités ou qualités entre lesquelles elle établit une «commensuration». Ainsi, c'est la dimension quantitative de la comparaison qui semble avoir la primauté.

Pourtant le rapport privilégié de comme avec le degré soulève des contestations : pour Sandfeld (1965 : 424), par exemple, comme sert d'abord à « désigner la conformité en ce qui concerne la manière dont s'accomplit une action ou se présente un fait » (nous soulignons). Dans leur étude comparative de comme et des tours corrélatifs en que, Fournier \& Fuchs (2007) semblent confirmer cette idée : 
Analogues en tant qu'opérateurs de chevillage, les deux adverbes s'opposent par leur sémantisme : que est à la base un marqueur de degré et comme est un marqueur de modus; ainsi la comparaison avec que est prototypiquement une comparaison quantitative (par identification d'un degré indéterminé), et la comparaison avec comme est prototypiquement qualitative (par identification d'un modus indéterminé). (Fournier \& Fuchs, Ibid. : 102)

En outre, l'utilisation assez contrainte du premier s'oppose à la souplesse du second, dont la portée variable est propice à de nombreux effets de sens conduisant jusqu'aux limites de la comparaison. Cette souplesse d'emploi ressort tout autant si l'on considère plus largement le système de la comparaison, ce que font Hadermann, Pierrard \& Van Raemdonck (2006), qui s’intéressent conjointement aux introducteurs de propositions comparatives, les « marqueurs d'identité similative » ainsi que, comme, de même que, etc.

Plus spécifiquement, d'autres travaux s'attachent aux seules comparatives en comme, avec l'objectif de rendre compte des régularités à la fois syntaxiques et sémantiques correspondant à des «types » de constructions identifiés. En s'intéressant à l'articulation des plans syntaxique et sémantique, Desmets (2001) montre comment le sens de manière associé à comme, alors introducteur d'une relative adverbiale sans antécédent ${ }^{12}$, produit l'effet de sens comparatif portant sur des qualités. En cherchant à proposer une analyse unifiée du fonctionnement comparatif de comme, l'étude de Desmets fait dériver les différentes valeurs interprétatives (manière, degré, qualia, valeur de vérité, etc.) du type d'items confrontés par la comparaison : entités, procès, propositions, etc. Prônant également une unification des comparatives en comme, Moline (2006) s'élève contre la sous-spécification proposée par Leroy (2003, 2004), qui suggère d'isoler le type des comparaisons à parangon, du type «sale comme un peigne ». Pour Leroy, en effet, ces constructions ne sont comparatives qu'en apparence, faisant usage d'un comparant stéréotypé pour signifier en réalité le haut degré. Pour des motifs essentiellement méthodologiques, Moline (2006:19) s'inscrit en faux contre une telle analyse et, afin de conserver une "description syntaxique unifiée des comparatives en comme», elle choisit de faire passer au second plan l'effet de sens de haut degré lié à certains comparants.

Bien que ce débat semble consacré à des aspects très particuliers de la comparaison, il soulève la question difficile de la délimitation de l'objet en linguistique: faut-il limiter la description aux propriétés inhérentes au mot ou au morphème (si tant est qu'on les ait identifiées), ou bien faut-il au contraire proposer une typologie des macrostructures dans lesquelles ce morphème apparaît ? Illustrant cette dernière approche, les travaux de Lambrecht $(1995,2004)$, reprenant la suite de Fillmore \& Kay, s'attachent à identifier et à décrire des constructions grammaticales ${ }^{13}$ intégrant le morphème comme, c'est-à-dire des

12 Autrement dit, une relative ayant pour antécédent non pas un nom mais une relation prédicative. Cette analyse va dans le sens de Sandfeld (1965: 428), Moignet (1981 : 198), Monneret \& Rioul, 1999 : 263). Mais aucun d'entre eux ne se résolvait à faire explicitement état de "relative».

${ }^{13}$ Cf. la contribution de Tobback et Defrancq dans ce volume. 
configuration[s] morphosyntaxique[s] et prosodique[s] dont la forme et l'interprétation ne peuvent être entièrement expliquées à partir des autres propriétés grammaticales du langage, et qui requièr[ent] donc une description indépendante (Lambrecht, 1995 : 187 , nous traduisons).

Le travail sur des structures de grande dimension nécessite de déborder le cadre morphosyntaxique, aussi Lambrecht prend-il également en compte les propriétés pragmatiques et informationnelles de son objet. Néanmoins, l'identification de telles constructions ne présente un réel intérêt que si les effets de sens non-compositionnels sont motivés, c'est-à-dire reliés à d'autres régularités de la langue. C'est ainsi que Lambrecht s'attache à confronter les constructions du type «c'est joli, comme endroit », à d'autres constructions fréquentes du français oral contemporain, qui entrent en concurrence dans certains contextes précis. On voit ainsi se dessiner la possibilité d'une systématique des unités de discours qui articule très clairement les déterminations globales (contexte, macrostructures) et les phénomènes analysés localement (microsyntaxe).

\section{Ouvertures}

Les points abordés dans ces quelques pages n'ont pas l'ambition de constituer la synthèse complète des études relative au morphème comme : la brève étude qui vient d'être proposée ne vise qu'à poser un regard prospectif sur les problématisations à venir.

En premier lieu, la question de la polysémie ou de l'homonymie de comme semble devoir se résoudre à l'avantage de la première hypothèse, ce à quoi l'influence du paradigme cognitiviste et, avant cela, celle de la psychomécanique du langage, ne sont sans doute pas étrangères. Mais les théories mentalistes de comme ${ }^{14}$ doivent proposer une description plus précise du signifié fondamental de comme au sein du lexique mental mais aussi une simulation du fonctionnement cognitif du morphème. Quoi qu'il en soit, faute de données neurolinguistiques, cette question relève encore d'un parti pris méthodologique et ne se résoudra sans doute pas par l'observation empirique des faits linguistiques. Il reste que l'hypothèse polysémique apparaît en outre plus élégante par son économie - et par le fait qu'elle masque partiellement (ou du moins qu'elle retarde) la répartition des occurrences en classes artificielles.

Ensuite, le problème de la catégorie de comme ne sera sans doute pas tranché sans une résistance des tenants de la nomenclature traditionnelle des parties du discours. Fort heureusement, la possibilité d'une autre typologie demeure, une typologie ayant acquis les propriétés de déformabilité nécessaires à la prise en compte d'objets complexes. Mais acceptera-t-on de parler de mots plus ou moins conjonctifs ou prépositionnels? Se résignera-t-on aux comportements adverbiaux ? Rien n'est moins sûr. En somme, la remarque très pertinente de Culioli peut paraître optimiste :

Pendant longtemps et de façon, au reste, inévitable, on a insisté sur les propriétés classificatoires des phénomènes linguistiques. D'où un travail fondé sur des étiquettes, des propriétés en tout ou rien, des identifications stables et prises dans des

${ }^{14}$ Entre autres Léard \& Pierrard (2003) et Fuchs \& Le Goffic (2005). 
hiérarchies rigides [...]. Grâce à une solide division disciplinaire (phonétique ; syntaxe; sémantique; pragmatique), on n'avait pas à aborder de front la question de la complexité des phénomènes. (Culioli, $1990: 128$ )

Pourtant, la plupart des études récentes du morphème comme semblent avoir pris acte de ces nouvelles exigences, que ce soit par l'intermédiaire de classes aux contours flous ou à travers la notion de proforme, dont les traits définitoires prototypiques relèvent de niveaux d'analyse différents (syntaxe, sémantique). Mais il n'est pas exclu que cette classification prenne pour objets les contextes où apparaissent les morphèmes, et non les morphèmes eux-mêmes : la classification réductionniste des unités lexicales doit être complétée par une typologie holiste des structures fondée sur la mise en évidences de contraintes globales correspondant aux différents paliers de complexité (Rastier 2001).

\section{RÉFÉRENCES BIBLIOGRAPHIQUES}

Arrivé, M. et al. (1986), La Grammaire d'aujourd'bui, Paris, Flammarion.

BÉCHADE, H. (1996), Grammaire française, Paris, PUF.

Charaudeau P. (1992), Grammaire du sens et de l'expression, Paris, Hachette.

Culioli, A. (1990) [1986], «Stabilité et déformabilité en linguistique », Étude de Lettres, Langages et connaissances, Lausanne, repris dans Pour une linguistique de l'énonciation, t. I, Opérations et représentations, Paris-Gap, Ophrys.

Damourette, J. \& Pichon, É. (1953), Des mots à la pensée, Essai de Grammaire de la Langue Française, Paris, D'Artrey.

DeSmets, M. (2001), Les typages de la phrase en HPSG : le cas des phrases en "comme", Thèse de doctorat, Paris X - Nanterre.

Flaux N. \& Moline E. (2008), «Constructions en comme : homonymie ou polysémie ? Introduction », Langue française, Paris, Larousse.

FOURNIER N. \& FUCHS C. (2007), "Que et comme marqueurs de comparaison », Les mots qu- du français, Lexique, 18, Villeneuve d'Ascq, Presses du Septentrion.

Fuchs, C. \& Le Goffic, P. (2005), «La polysémie de comme » in Soutet O. (éd), La Polysémie, Paris, PUPS, p. 267-291

Gardes-Tamine, J. (1998), La Grammaire, 2- Syntaxe, Paris, Armand Colin.

Grevisse, M. \& Goosse, A. (1993), Le Bon Usage, 13e édition, Bruxelles-Leuwen, De BoeckDuculot.

Hadermann, P., Pierrard, M. \& Van Raemdonck, D. (2006), «Les marqueurs d'identité : subordonnants, coordonnants ou corrélateurs », Faits de langue 28, Paris, Ophrys, p. 133-145.

LAMbrecht, K. (1995), "Compositional vs. constructional meaning: the case of French comme N », in T. Galloway \& Simons, M. (eds.), Proceedings of the 5th SALT Conference, Cornell University, p.186-203. 
Lauwers, P. \& Neveu, F. (2007), «La notion de "tradition grammaticale" et son usage en linguistique française », Langages, 167, Paris, Larousse, p. 7-26.

LE GOFFIC, P. (1991), «Comme, adverbe connecteur intégratif : éléments pour une description », Travaux Linguistiques du CERLICO 4, P.U.R., p. 11-31.

LE Goffic, P. (1993), Grammaire de la Phrase française, Paris, Hachette.

LÉARD, J.-M. \& PierRARD, M. (2003), «L'analyse de comme : le centre et la périphérie », in Hadermann P., Van Scijcke A. \& Berré M. (eds), La syntaxe raisonnée, Mélanges de linguistique générale et française offerts à Annie Boone, Bruxelles, Duculot, p. 203-234.

LEROY S. (2003), «Les constructions comparatives intensives dans Illusions perdues», L'information grammaticale, 99, Louvain, Peeters, p. 39-42.

LEROY S. (2004), «Sale comme un peigne et méchant comme une teigne. Quelques remarques sur les comparaisons à parangon », Travaux linguistiques du CERLICO 17, P.U.R., p. 297-308.

Moline, E. (2006), «Belle comme un cour/Belle comme sa scur. Pour une description unifiée des comparatives en comme », L'information grammaticale, 111, Louvain, Peeters, p. 14-20.

Monneret, Ph. \& Rioul, R. (1999), Questions de syntaxe francaise, Paris, PUF.

MulLER, C. (1996), La subordination en français. Le schème corrélatif, Paris, Armand Colin.

NøLKe, H. (1993), Le regard du locuteur, Paris, Kimé.

Pierrard, M. (1999a), «Grammaticalisation et contexte : l'extension des emplois de comme », Revue de Sémantique et de pragmatique, 6, Orléans, PUO, p. 133-144.

Pierrard, M. (1999b), "Comme, relateur de prédicats», Cabiers de l'institut de linguistique de Lomvain 25-2, Louvain-la-neuve, Institut de linguistique, p. 153-168.

- (2002a), « Grammaticalisation et restructuration fonctionnelle : comme et la subordination », in Lagorgette D. et Larrivée P. (eds), Représentation du sens linguistique, Lincom Europa, p. 293-307.

- (2002b), «Comme préposition ? Observations sur le statut catégoriel des prépositions et des conjonctions ", Travaux de linguistique, 44, Bruxelles, De Boeck, p. 69-78.

Pierrard, M. \& LeARD, J.-M. (2004), « Comme : comparaison et haut degré », Travaux linguistiques $d u$ CERLICO, 17, Rennes, P.U.R., p. 269-286.

- (2005), «Proformes indéfinies et expressions indéfinies : à propos du lien entre indéfinition et prédication », in Corblin F., Ferrando S. \& Kupferman (eds), Indéfini et prédication, Paris, PUPS, p. 493-506.

Rastier, F. (2001), Arts et sciences du texte, Paris, P.U.F.

Riegel, M., Pellat, J.C. \& Rioul R. (1994), Grammaire méthodique du français, Paris, P.U.F.

RIVARA, R. (1990), Le système de la comparaison, Paris, Éd. de Minuit.

Wagner \& Pinchon (1993 [1e éd. 1962]), Grammaire du français classique et moderne, Paris, Hachette.

Wilmet, M. (1997), Grammaire critique du français, Paris-Bruxelles, Hachette-Duculot. 
